\title{
Color superconductivity and quark stars
}

\author{
M. Malheiro, ${ }^{a}{ }^{b *}$ L. P. Linares,${ }^{b \dagger}$ M. Fiolhais, ${ }^{c}$ A. Taurines,${ }^{d} \ddagger$ \\ ${ }^{a}$ Instituto Tecnológico de Aeronáutica, \\ CTA, 12-228-900, São José dos Campos, Brazil \\ ${ }^{\mathrm{b}}$ Instituto de Física, Universidade Federal Fluminense, \\ 24-210-320, Niterói, Brazil \\ ${ }^{\mathrm{c}}$ Departamento de Física, Centro de Física Computacional, \\ Universidade de Coimbra, P-3004-516 Coimbra, Portugal \\ ${ }^{\mathrm{d}}$ Department of Physics, University of Wales Swansea, \\ Singleton Park, Swansea, SA2 8PP, United Kingdom
}

The search for new phases of strange quark matter inside neutron stars has recently received a lot of attention since it has been shown that the attractive nature of the one gluon exchange interaction in QCD may produce a superconducting phase in quark matter. We study an extended version of the Chromodielectric model with a BCS quark pairing implemented, and analyze the superconducting color flavor locked phase. We show that the inclusion in the free energy density of a negative term of the diquark condensate ensures the stability of quark matter. We explore the implications of our results in the structure of compact quark stars and explicitly show that CFL stars can be absolutely stable and more compact than strange stars.

\section{INTRODUCTION}

The QCD attractive interaction of quarks close to the Fermi surface may lead to the formation of Cooper pairs which condense since they are bosons. The condensates generate gaps in the fermionic spectra which can be interpreted as the masses of these quasi-quarks. Since quark pairs in QCD cannot be color singlets, the color symmetry must be broken and this phenomenon is known as color superconductivity. For three flavors of massless quarks, a new phenomenon occurs as the condensate of quark pairs is formed: since the Cooper pairs cannot be flavor isosinglets, both the chiral symmetry and the color symmetry are broken. The condensate formed through the attractive channel of the one gluon exchange exhibits what it is known in literature as Color Flavour Locking (CFL) [1]. In this work we are going to investigate, using the Chromodieletric model (CDM) [2], high density strange quark matter in the CFL phase [3]. We choose this model, where quarks interact with effective mesons fields, since its chiral symmetry

\footnotetext{
${ }^{*} \mathrm{CNPq}$.

${ }^{\dagger}$ Capes.

${ }^{\ddagger} \mathrm{CNPq}$, FAPESP
} 
is spontaneously broken, generating dynamical masses for quarks (a common feature with NJL models), and since it confines at low energies.

Moreover, in the framework of the same model, a study of the unpaired three flavor quark matter in $\beta$ equilibrium has been carried on [4] and problems with the stability of this strange matter at high densities have been found. These problems are solved through the introduction of a quark pairing interaction, since the strange matter in the superconducting phase has a lower energy per particle than in the unpaired quark phase. The CDM model will be used with a quartic potential.

The superconducting color phase of quark matter may have interesting consequences in astrophysics. In the normal unpaired quark matter, it was shown that very small compact stars with radius less than $8 \mathrm{~km}$ are metastable [4]. We will show that it is possible to obtain CFL compact stars, with a maximum mass $M / M_{\odot}=1.43$ and radius $R \sim 7.5 \mathrm{~km}$, where the quark matter is stable $(\varepsilon<\rho M)$. This result seems to indicate that pure quark stars, if they really exist in nature, are likely to be in the CFL phase.

\section{THE CFL PHASE IN THE CHROMODIELECTRIC MODEL}

The Lagrangian density of the CDM extended to the strange sector can be written as [2]

$$
\begin{aligned}
\mathcal{L} & =\mathrm{i} \bar{\psi} \gamma^{\mu} \partial_{\mu} \psi+\frac{1}{2}\left(\partial_{\mu} \sigma \partial^{\mu} \sigma+\partial_{\mu} \vec{\pi} \cdot \partial^{\mu} \vec{\pi}\right)-W(\sigma, \vec{\pi})+ \\
& +\frac{g}{\chi} \bar{\psi}\left(\sigma+\mathrm{i} \vec{\tau} \cdot \vec{\pi} \gamma_{5}\right) \psi+\frac{g_{s}}{\chi} \bar{\psi}_{s} \psi_{s}+\frac{1}{2} \partial_{\mu} \chi \partial^{\mu} \chi-U(\chi) .
\end{aligned}
$$

The first and second terms describe the quark and meson kinetic energies, respectively, and the third one the chiral meson self-interaction (Mexican hat potential). The fourth and fifth terms in (1) describe the meson-quark interaction: the former refers only to the two light quark flavors ( $u$ and $d$ ) and the latter to the strange quark $s$. The last two terms in the Lagrangian density (1) refer to the dynamical confining $\chi$ field, namely to its kinetic (sixth term) and potential, $U(\chi)$, energies. We use a CDM version with the following quartic potential for the $\chi$ field:

$U(\chi)=\frac{1}{2} m_{\chi}^{2} \chi^{2} \times\left[1+\left(\frac{8 \eta^{4}}{\gamma^{2}}-2\right)\left(\frac{\chi}{\gamma m_{\chi}}\right)+\left(1-\frac{6 \eta^{4}}{\gamma^{2}}\right)\left(\frac{\chi}{\gamma m_{\chi}}\right)^{2}\right]$

where $m_{\chi}$ is the mass of the $\chi$ field. The potential has an absolute minimum at $\chi=0$. The other two parameters acquire a simple meaning when the potential is written as in Eq. (2): $\gamma$ defines the position of the second (local) minimum, located at $\chi=\gamma m_{\chi}$, and $\eta$ the value of the potential at this minimum: $U\left(\gamma m_{\chi}\right)=\left(\eta m_{\chi}\right)^{4}$. The range for $\eta$ is the same as in [4].

The meson-quark interaction terms generate dynamical quark masses, being all different (see [5] for details). The parameters that better reproduce the nucleon properties in this model are the coupling constant, $g=0.023 \mathrm{GeV}$, the $\chi$ field mass, $m_{\chi}=1.7 \mathrm{GeV}$ and $\gamma=0.2$, which we keep fixed at this value as in $[4,5]$.

We now extend the CDM to include the diquark condensate. The superconducting CFL phase appears when an attractive term responsible for the quark pairing is introduced. This term reads $-3\left(\frac{\Delta \mu}{\pi}\right)^{2}$ and its effect is to decrease the free energy of the system. The gap energy, $\Delta$, in principle, depends on the quark chemical potential, $\mu$, in the range of neutron star densities $(\mu$ between 400 to $500 \mathrm{MeV}$ ), but we take it as a constant. 
At zero temperature, the grand-potential divided by the system volume is $\Omega=\varepsilon-\mu \rho$ where $\varepsilon$ is the energy density, $\mu$ is the chemical potential and $\rho$ the particle density. The grand-potential density of CDM in the superconducting color phase (CFL), reads

$\Omega_{\mathrm{CFL}}=\varepsilon_{k}-\frac{1}{\pi^{2}} \sum_{i=u, d, s} \mu_{i} k_{i}^{3}-3\left(\frac{\Delta \mu}{\pi}\right)^{2}+U(\chi)+W(\sigma, \vec{\pi})$

where

$\varepsilon_{k}=\frac{3}{\pi^{2}} \sum_{i=u, d, s} \int_{0}^{k_{i}}\left(k^{2} \sqrt{\left(k^{2}+m_{i}^{2}\right)}\right) d k$

is the kinetic energy for a relativistic quark gas. The quark masses, $m_{i}$, are expressed in terms of the meson fields and $\alpha=6$. The quark matter energy density can now be written as

$\varepsilon=\varepsilon_{k}-\frac{1}{\pi^{2}} \sum_{i=u, d, s} \mu_{i} k_{i}^{3}-3\left(\frac{\Delta \mu}{\pi}\right)^{2}+\sum_{i=u, d, s} \mu_{i} \rho_{i}+U(\chi)+W(\sigma, \vec{\pi})$.

To impose the local charge neutrality the Fermi momenta $\left(k_{i}=\sqrt{\mu_{i}^{2}-m_{i}^{2}}\right)$ of all quarks should be equal, $k_{f}=k_{u}=k_{d}=k_{s}=2 \mu-\sqrt{\mu^{2}+\frac{m_{s}^{2}}{3}}$ (we define the quark chemical potential, $\mu$, through $3 \mu=\sum_{i=u, d, s} \mu_{i}$ and admit that the $u$ and $d$ quark masses are very small).

When we take the derivative of the grand-potential (3) with respect to the chemical potential of each quark, $\mu_{i}$, we obtain the same quark density for $u, d$ and $s$ if all quarks have the same Fermi momentum in the CFL phase. Thus, if we use the definition of the above average quark chemical potential, $\mu$, the baryonic density $\rho$ is given by $\rho=\rho_{u}=\rho_{d}=\rho_{s}=\frac{1}{\pi^{2}}\left(k_{f}^{3}+2 \Delta^{2} \mu\right)$ and the energy density of the CFL phase of strange quark matter (5) can be cast in the form $\varepsilon=\varepsilon_{k}+3\left(\frac{\Delta \mu}{\pi}\right)^{2}+U(\chi)+W(\sigma, \pi)$, where $\varepsilon_{k}$ is given by Eq. (4).

\section{RESULTS AND CONCLUSIONS}

As for the strange quark matter without pairing [4], the model accommodates two solutions: one for small values of $\chi$ (solution I) and the other one for large values of this field, near the second minimum of the confining potential $\chi \sim \gamma m_{\chi}$ (solution II). In Fig. 1 - left, the energy per particle for the solution II is shown as a function of the baryonic density, for various pairing parameters. As we observe, increasing of the pairing interaction $\Delta$ lowers the energy per particle, leading to a more stable quark matter. In the case of solution $\mathrm{I}(U(\chi) \sim 0)$, the strange matter is stable only for $\Delta<65 \mathrm{MeV}$. This happens because only for these values of $\Delta$, the pressure vanishes producing a minimum in Fig. 1 - right. For larger values of the pairing, the energy per particle does not show a minimum, and the matter collapses for low densities. In solution I, the values for the $\chi$ field are very small and the dynamical quark masses are large. This collapse indicates that the superconducting phase of massive quarks is not favored.

One motivation for the present work is to investigate whether bare quark stars in the CFL phase are absolutely stable, and how compact they are in comparison with strange stars made up of unpaired quark matter in $\beta$ equilibrium. To address this issue, we solved the OppenheimerVolkov equation for relativistic stars and present in Table 1 the results for the maximum mass, $M$, of CFL strange stars (in units of the solar mass, $M_{\odot}$ ), the radius, $R$, and the central energy density, $\varepsilon_{\mathrm{c}}$, for different values of $\eta$ (all yielding a stable quark matter). 

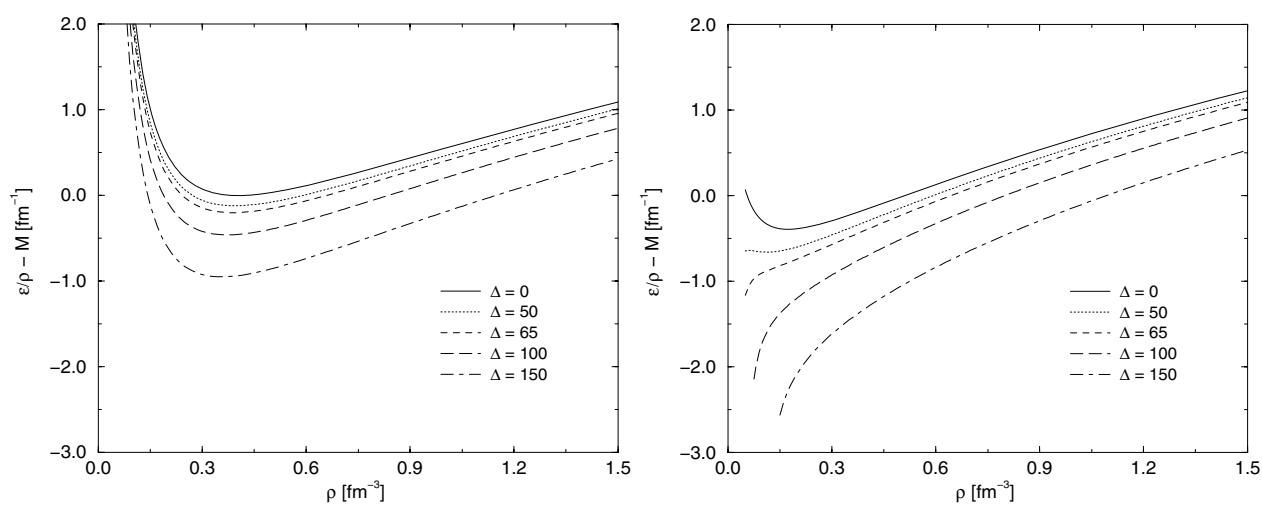

Figure 1. Energy per particle as a function of the baryonic density with $\eta=0.0966$ and different $\Delta$ pairing interaction for the solution II (left) and for solution I (right).

Table 1

\begin{tabular}{|c|c|c|c|c|}
\hline$\eta$ & $\Delta[\mathrm{MeV}]$ & $M\left[M_{\odot}\right]$ & $R[\mathrm{~km}]$ & $\varepsilon_{\mathrm{c}}\left[\mathrm{fm}^{-4}\right]$ \\
\hline 0.0966 & 0 & 1.56 & 8.48 & 9.62 \\
\cline { 2 - 5 } & 100 & 1.81 & 9.61 & 7.18 \\
\cline { 2 - 5 } & 150 & 2.11 & 10.81 & 5.72 \\
\hline 0.1053 & 100 & 1.49 & 7.94 & 10.62 \\
\cline { 2 - 5 } & 150 & 1.72 & 8.89 & 8.46 \\
\hline 0.1137 & 150 & 1.43 & 7.45 & 11.87 \\
\hline
\end{tabular}

Values of the maximum mass $M$, radius $R$ and central density energy $\varepsilon_{\mathrm{c}}$ for the stable CFL star with different values of $\eta$ and $\Delta$.

As we can read from this table, relatively large maximum masses for CFL stars can be obtained. Most of the neutron stars have a mass $M \sim 1.4 M_{\odot}$. It is interesting that in the maximum limit of quark matter stability (large $\eta$, for $\Delta=150 \mathrm{MeV}$ ) the maximum mass is around this value but with a much too small radius - only $R \leq 8 \mathrm{~km}$ - in comparison with the radius range from 10 to $14 \mathrm{~km}$ of a typical neutron star. With the parameters considered in our model, the maximum limit for the potential energy and for the gap energy to have stable quark matter are $\eta=0.1137$ and $\Delta=150 \mathrm{MeV}$ respectively. This yields very compact CFL stars which are stable, something that is not possible to obtain in the case of the unpaired quark matter without facing the problem of the metastability [4].

\section{REFERENCES}

1. M. Alford, K. Rajagopal and F. Wilczek, Nucl. Phys. B 537 (1999) 433.

2. M. K. Banerjee, Prog. Part. Nucl. Phys. 31 (1993) 77, M. C. Birse, Prog. Part. Nucl. Phys. 25 (1990) 1.

3. L. P. Linares Color superconducting phase in the chromodielectric model and applications to quark stars, Thesis (in Portuguese), Universidade Federal Fluminense, Niterói, 2005 (unpublished).

4. M. Malheiro, M. Fiolhais and A. R. Taurines, J. Phys. G 29 (2003) 1045.

5. A. Drago, M. Fiolhais and U. Tambini, Nucl. Phys. A 609 (1996) 488. 